\title{
On Filter $(\alpha)$-convergence and Exhaustiveness of Function Nets in Lattice Groups and Applications
}

\author{
Antonio Boccuto $^{1} \&$ Xenofon Dimitriou ${ }^{2}$ \\ ${ }^{1}$ Dipartimento di Matematica e Informatica, via Vanvitelli, 1 I-06123 Perugia, Italy \\ ${ }^{2}$ Department of Mathematics, University of Athens, Panepistimiopolis, Athens 15784, Greece \\ Correspondence: Antonio Boccuto, Dipartimento di Matematica e Informatica, via Vanvitelli, 1 I-06123 Perugia, \\ Italy. E-mail: antonio.boccuto@unipg.it
}

Received: March 16, 2015 Accepted: March 22, 2015 Online Published: March 27, 2015

doi:10.5539/jmr.v7n2p56 URL: http://dx.doi.org/10.5539/jmr.v7n2p56

\begin{abstract}
We consider (strong uniform) continuity of the limit of a pointwise convergent net of lattice group-valued functions, (strong weak) exhaustiveness and (strong) $(\alpha)$-convergence with respect to a pair of filters, which in the setting of nets are more natural than the corresponding notions formulated with respect to a single filter. Some comparison results are given between such concepts, in connection with suitable properties of filters. Moreover, some modes of filter (strong uniform) continuity for lattice group-valued functions are investigated, giving some characterization. As an application, we get some Ascoli-type theorem in an abstract setting, extending earlier results to the context of filter $(\alpha)$-convergence. Furthermore, we pose some open problems.
\end{abstract}

Keywords: lattice group, (strong uniform) continuity, (strong weak) filter exhaustiveness, (strong) filter $(\alpha)$-convergence, filter compactness, filter closedness.

2010 A. M. S. Mathematical Classifications: Primary: 26E50, 28B10, 28B15, 40A35, 46G10, 54A20, 54A40. Secondary: 22A10, 28A05, 40G15, 46G12, 54H11, 54H12.

\section{Introduction}

In this paper we deal with (strong) filter $(\alpha)$-convergence for nets of lattice group-valued functions defined on abstract topological spaces, (strong) filter exhaustiveness and (strong uniform) continuity. The concept of strong uniform continuity is related to the problem of finding a topology finer than that of pointwise convergence in connection with preservation of continuity of the limit function, and was introduced in Beer and Levi (2009) and recently investigated by several authors (see also Caserta, Di Maio and Holá (2010), Caserta, Di Maio and Kočinac (2011) and the literature therein). The concept of $(\alpha)$-convergence or continuous convergence of real-valued function sequences has been known in the literature since the beginning of the last century (see also Carathéodory (1929) and Hahn (1922)). In Zygmund (1968) it is proved that the linear means of the trigonometric series of a real-valued function $f$, continuous on $[0,2 \pi]$ and summable with respect to a suitable positive kernel, $(\alpha)$-converge to $f$. In general, (strong) filter $(\alpha)$-convergence for function nets is different from the corresponding notion given for sequences, since the set of the indexes of the function net can be larger than the set on which these functions are defined (see also Gregoriades and Papanastassiou (2008)). We give a characterization of (strong) $(\alpha)$-convergence in terms of (strong) filter exhaustiveness, without requiring special hypotheses on the involved filter, like in the classical case (see also Boccuto, Dimitriou, Papanastassiou and Wilczyński (2011)). This leads to considering a concept of (weak) filter exhaustiveness with respect to a pair of filters. We give some characterization of (strong uniform) continuity of the limit function and investigate some modes of (strong uniform) continuity of lattice group-valued functions with respect to a pair of filters in the context of nets, extending some results proved in Baláž, Červeňanskij, Kostyrko and Šalát (2002). Moreover we give some comparison results between filter ( $\alpha$ )convergence with respect to a pair of filters and usual $(\alpha)$-convergence with respect to one filter and between filter exhaustiveness with respect to a pair of filters and usual exhaustiveness with respect to a single filter, and illustrate some differences between strong filter $(\alpha)$-convergence and filter $(\alpha)$-convergence, and between (strong) exhaustiveness and (strong) weak exhaustiveness. Some related results have been proved in Caserta, Di Maio and 
Holá (2010), Gregoriades and Papanastassiou (2008) for the classical case, in Caserta, Di Maio and Kočinac (2011) for the statistical convergence and in Athanassiadou, Boccuto, Dimitriou and Papanastassiou (2012), Athanassiadou, Dimitriou, Papachristodoulos and Papanastassiou (2012), Boccuto and Dimitriou (2011, 2014), Boccuto, Dimitriou, Papanastassiou and Wilczyński (2011) in connection with filter/ideal continuous convergence for function sequences, where exhaustiveness is intended with respect to one filter. In this setting, we investigate also some property of the filter $\mathcal{F}_{\text {st }}$ of all subsets of $\mathbb{N}$ having asymptotic density one. As an application of filter $(\alpha)$ convergence and the related notions of filter exhaustiveness, we give an abstract Ascoli-type theorem, where the involved concepts of closedness and compactness are formulated in terms of suitable convergences of nets, without assuming necessarily the existence of a structure of Hausdorff topology. In this framework, we extend some results proved in Athanassiadou, Boccuto, Dimitriou and Papanastassiou (2012), Boccuto and Dimitriou (2014), Gregoriades and Papanastassiou (2008) and Kelley (1955). Furthermore, we consider filter (weak) exhaustiveness and filter $(\alpha)$-convergence with respect to a triple of filters, in relation with the problem of finding necessary/sufficient conditions for filter continuity of the limit of a function net. Furthermore, we consider filter (weak) exhaustiveness and filter $(\alpha)$-convergence with respect to a triple of filters, in relation with the problem of finding necessary/sufficient conditions for filter continuity of the limit of a function net. Finally, we pose some open problems.

\section{Preliminaries}

We now give the notions, which will be useful in the sequel in proving our main results.

A lattice group (or $(\ell)$-group) is an abelian partially ordered group $R=(R,+, \leq)$ closed with respect to the operations of finite supremum and finite infimum, and such that $a+c \leq b+c$ whenever $a, b, c \in R$ and $a \leq b$. A lattice group $R$ is said to be Dedekind complete iff every nonempty subset of $R$, bounded from above, admits supremum in $R$ (see also Boccuto and Dimitriou (2015)).

Throughout the paper, we denote by $R$ a Dedekind complete $(\ell)$-group. A sequence $\left(\sigma_{p}\right)_{p}$ in $R$ is called an $(O)$ sequence iff it is decreasing and $\bigwedge_{p} \sigma_{p}=0$. A net $\left(x_{\lambda}\right)_{\lambda \in \Lambda}$ in $R$ is order convergent (or $(O)$-convergent) to $x \in R$ iff there exists an $(O)$-sequence $\left(\sigma_{p}\right)_{p}$ in $R$ such that for every $p \in \mathbb{N}$ there exists $\lambda \in \Lambda$ with $\left|x_{\zeta}-x\right| \leq \sigma_{p}$ for all $\zeta \in \Lambda, \zeta \geq \lambda$, and in this case we will write $(O) \lim _{\lambda \in \Lambda} x_{\lambda}=x$.

If $X$ is a topological space and $x \in X$, then we say that a function $f: X \rightarrow R$ is continuous at $x$ iff there is an $(O)$-sequence $\left(\sigma_{p}\right)_{p}$ (depending on $x$ ) with the property that for each $p \in \mathbb{N}$ and $x \in X$ there exists a neighborhood $U_{x}$ of $x$ with $|f(x)-f(z)| \leq \sigma_{p}$ whenever $z \in U_{x}$ (see also Boccuto, Dimitriou, Papanastassiou and Wilczyński (2014))

Let $X=(X, \mathcal{D})$ be a uniform space, where $\mathcal{D}$ denotes the set of the entourages. If $\emptyset \neq B \subset X$, then a function $f: X \rightarrow R$ is strongly uniformly continuous on $B$ iff there exists an $(O)$-sequence $\left(\sigma_{p}\right)_{p}$ such that for every $p \in \mathbb{N}$ there is $D \in \mathcal{D}$ with $|f(\beta)-f(x)| \leq \sigma_{p}$ whenever $x \in X, \beta \in B$ and $(x, \beta) \in D$.

Let $(\Lambda, \geq)$ be a directed set. A filter $\mathcal{F}$ of $\Lambda$ is $(\Lambda)$-free iff $\mathcal{F} \neq \emptyset, \emptyset \notin \mathcal{F}$ and $M_{\lambda} \in \mathcal{F}$ for each $\lambda \in \Lambda$, where $M_{\lambda}:=\{\zeta \in \Lambda: \zeta \geq \lambda\}$.

We denote by $\mathcal{F}_{\text {cofin }}$ the filter of all subsets of $\mathbb{N}$ whose complement is finite, by $\mathcal{I}_{\text {fin }}$ the family of all finite subsets of $\mathbb{N}$, by $\mathcal{F}_{\text {st }}$ the filter of all subsets of $\mathbb{N}$ having asymptotic density 1 , by $\mathcal{I}_{\text {st }}$ the family of all subsets of $\mathbb{N}$ with asymptotic density 0 and by $\mathcal{F}_{\Lambda}$ the class $\left\{A \subset \Lambda\right.$ and $\left.A \supset M_{\lambda}: \lambda \in \Lambda\right\}$.

Some filters $\mathcal{F}$ of $\mathbb{N}$ have the property that there is a partition of the type $\mathbb{N}=\bigcup_{k=1}^{\infty} \Delta_{k}$, such that

$$
\mathcal{I}=\left\{A \subset \mathbb{N}: A \text { intersects at most a finite number of } \Delta_{k}{ }^{\prime} \mathrm{s}\right\},
$$

where $\mathcal{I}$ denotes the dual ideal of $\mathcal{F}$ (see also Boccuto, Das, Dimitriou and Papanastassiou (2012)).

Remarks 2.1. (a) Observe that the ideal $\mathcal{I}_{\text {fin }}$ satisfies condition (1): indeed it is enough to take $\Delta_{k}=\{k\}$ for each $k \in \mathbb{N}$.

(b) If $\mathcal{I}$ is as in (1), and $\left(A_{j}\right)_{j}$ is any sequence of subsets of $\mathbb{N}$, with $A_{j} \notin \mathcal{I}$ for all $j \in \mathbb{N}$, then there exists a disjoint sequence $\left(B_{j}\right)_{j}$ in $\mathcal{I}$, with $B_{j} \subset A_{j}$ for every $j \in \mathbb{N}$ and $\bigcup_{j=1}^{\infty} B_{j} \notin \mathcal{I}$ (see also Boccuto, Das, Dimitriou and 
Papanastassiou (2012), Remark 4.15 (b)).

(c) We now see that the ideal $\mathcal{I}_{\text {st }}$ does not fulfil condition (1). To this aim, it is enough to show that for every partition $\left(\Delta_{k}\right)_{k}$ of $\mathbb{N}$ there is a set belonging to $\mathcal{I}_{\text {st }}$ which intersects infinitely many $\Delta_{k}$ 's. Set $q(1)=1$ : there is $k_{1} \in \mathbb{N}$ with $1 \in \Delta_{k_{1}}$. At the second step, take a natural number $q(2)$ greater than $q(1)+3$ and belonging to $\Delta_{k_{2}}$, where $k_{2}$ is a suitable integer strictly greater than $k_{1}$. At the $n+1$-th step, if we have chosen $q(n)$, let $q(n+1)$ be an integer greater than $q(n)+2 n+1$ and belonging to $\Delta_{k_{n+1}}$, where $k_{1}<k_{2}<\ldots<k_{n}<k_{n+1}$. It is not difficult to check that the set $A=\{q(n): n \in \mathbb{N}\}$ has asymptotic density smaller or equal than that of the set of squares, that is 0 , and thus $A \in \mathcal{I}_{\text {st. }}$. Moreover, by construction, $A$ intersects infinitely many $\Delta_{k}$ 's.

Let $X$ be any Hausdorff topological space, $x \in X$ and $\mathcal{F}$ be a $(\Lambda)$-free filter of $\Lambda$. A net $\left(x_{\lambda}\right)_{\lambda \in \Lambda} \mathcal{F}$-converges to $x$ (shortly, $(\mathcal{F}) \lim _{\lambda \in \Lambda} x_{\lambda}=x$ or $(\mathcal{F}) \lim _{\lambda} x_{\lambda}=x$ ) iff $\left\{\lambda \in \Lambda: x_{\lambda} \in U\right\} \in \mathcal{F}$ for each neighborhood $U$ of $x$.

Let $\Xi$ be any nonempty set. A family $\left\{\left(x_{\lambda, \xi}\right)_{\lambda}: \xi \in \Xi\right\}$ in $R(R O \mathcal{F})$-converges to $\left\{x_{\xi}: \xi \in \Xi\right\}$ iff there is an $(O)$ sequence $\left(\sigma_{p}\right)_{p}$ such that for each $p \in \mathbb{N}$ and $\xi \in \Xi$ we get $\left\{\lambda \in \Lambda:\left|x_{\lambda, \xi}-x_{\xi}\right| \leq \sigma_{p}\right\} \in \mathcal{F}$. We say that the function net $f_{\lambda}: X \rightarrow R, \lambda \in \Lambda,(R O \mathcal{F})$-converges to $f: X \rightarrow R$ iff the family $\left\{\left(f_{\lambda}(x)\right)_{\lambda}: x \in X\right\}(R O \mathcal{F})$-converges to $\{f(x)$ : $x \in X\}$ (see also Boccuto and Dimitriou (2015), Boccuto, Dimitriou and Papanastassiou (2012a-b), Candeloro and Sambucini (2015)).

Let $(X, \mathcal{D})$ be a uniform space, $\emptyset \neq B \subset X, \Xi=(\Xi, \geq)$ be a directed set and $\mathcal{S}$ be a $(\Xi)$-free filter of $\Xi$. We say that the pair of nets $z_{\xi}, x_{\xi}, \xi \in \Xi$, satisfies condition H1) with respect to $\mathcal{S}$ iff $x_{\xi}, z_{\xi} \in B$ for each $\xi \in \Xi$, and for every $D \in \mathcal{D}$ there is a set $F \in \mathcal{S}$ with $\left(x_{\xi}, z_{\xi}\right) \in D$ whenever $\xi \in F$.

Let $\mathcal{S}$ and $\mathcal{F}$ be any two fixed $(\Xi)$-free filters of $\Xi$. A function $f: X \rightarrow R$ is said to be strongly $(\mathcal{S}, \mathcal{F})$-uniformly continuous on $B$ iff there is an $(O)$-sequence $\left(\sigma_{p}\right)_{p}$ in $R$ such that for every pair of nets $z_{\xi}, x_{\xi}, \xi \in \Xi$, satisfying condition $\mathrm{H} 1$ ) with respect to $\mathcal{S}$, we have:

H2) for each $p \in \mathbb{N}$ there is $F^{*} \in \mathcal{F}$ with $\left|f\left(x_{\xi}\right)-f\left(z_{\xi}\right)\right| \leq \sigma_{p}$ for each $\xi \in F^{*}$.

Let $X$ be any Hausdorff topological space. We say that $f: X \rightarrow R$ is $(\mathcal{S}, \mathcal{F})$-continuous at $x \in X$ iff there is an $(O)$-sequence $\left(\sigma_{p}\right)_{p}$ in $R$ such that, for every net $\left(x_{\xi}\right)_{\xi \in \Xi} \mathcal{S}$-convergent to $x$, the net $\left(f\left(x_{\xi}\right)\right)_{\xi \in \Xi}(R O \mathcal{F})$-converges to $f(x)$ with respect to $\left(\sigma_{p}\right)_{p}$.

Let $(X, \mathcal{D})$ be a uniform space and $\emptyset \neq B \subset X$. A net of functions $f_{\lambda}: X \rightarrow R, \lambda \in \Lambda$, is said to be strongly $\mathcal{F}$-exhaustive on $B$ iff there is an $(O)$-sequence $\left(\sigma_{p}\right)_{p}$ such that for any $p \in \mathbb{N}$ there exist $D \in \mathcal{D}$ and $A \in \mathcal{F}$ such that for each $\lambda \in A$ and $x \in X, \beta \in B$ with $(x, \beta) \in D$ we have $\left|f_{\lambda}(x)-f_{\lambda}(\beta)\right| \leq \sigma_{p}$.

We say that a net $f_{\lambda}: X \rightarrow R, \lambda \in \Lambda$, is strongly weakly $\mathcal{F}$-exhaustive on $B$ iff there is an $(O)$-sequence $\left(\sigma_{p}\right)_{p}$ such that for each $p \in \mathbb{N}$ there is $D \in \mathcal{D}$ such that, for every $x \in X$ and $\beta \in B$ with $(x, \beta) \in D$, there is $A \in \mathcal{F}$ (depending on $x$ and $\beta$ ) with $\left|f_{\lambda}(x)-f_{\lambda}(\beta)\right| \leq \sigma_{p}$ whenever $\lambda \in A$.

Let $x \in X$. We say that a net $f_{\lambda}: X \rightarrow R, \lambda \in \Lambda$, is $\mathcal{F}$-exhaustive at $x$ iff there is an $(O)$-sequence $\left(\sigma_{p}\right)_{p}$ with the property that for any $p \in \mathbb{N}$ there exist a neighborhood $U$ of $x$ and a set $A \in \mathcal{F}$ such that for each $\lambda \in A$ and $z \in U$ we have $\left|f_{\lambda}(z)-f_{\lambda}(x)\right| \leq \sigma_{p}$.

A net $f_{\lambda}: X \rightarrow R, \lambda \in \Lambda$, is weakly $\mathcal{F}$-exhaustive at $x$ iff there is an $(O)$-sequence $\left(\sigma_{p}\right)_{p}$ such that for each $p \in \mathbb{N}$ there is a neighborhood $U$ of $x$ with the property that for any $z \in U$ there is $A_{z} \in \mathcal{F}$ with $\left|f_{\lambda}(z)-f_{\lambda}(x)\right| \leq \sigma_{p}$ whenever $\lambda \in A_{z}$.

When we deal with $(\alpha)$-convergence for nets of functions, it is advisable to consider (strong weak) exhaustiveness with respect to a pair of filters.

Let $\mathcal{S}$ and $\mathcal{F}$ be as above, $(X, \mathcal{D})$ be a uniform space and $\emptyset \neq B \subset X$. A net $f_{\lambda}: X \rightarrow R, \lambda \in \Lambda$, is said to be strongly $(\mathcal{S}, \mathcal{F})$-exhaustive on $B$ iff there exists an $(O)$-sequence $\left(\sigma_{p}\right)_{p}$ in $R$ such that, for every pair of nets $x_{\xi}, z_{\xi}$, $\xi \in \Xi$, satisfying H1) with respect to $\mathcal{S}$ and for any $p \in \mathbb{N}$ there are $S \in \mathcal{S}, F \in \mathcal{F}$ with $\left|f_{\lambda}\left(x_{\xi}\right)-f_{\lambda}\left(z_{\xi}\right)\right| \leq \sigma_{p}$ for every $\xi \in S$ and $\lambda \in F$.

The net $f_{\lambda}: X \rightarrow R, \lambda \in \Lambda$, is strongly weakly $(\mathcal{S}, \mathcal{F})$-exhaustive on $B$ iff there exists an $(O)$-sequence $\left(\sigma_{p}\right)_{p}$ in $R$ such that, for each pair of nets $x_{\xi}, z_{\xi}, \xi \in \Xi$, satisfying H1) with respect to $\mathcal{S}$ and for every $p \in \mathbb{N}$ there is a set $S \in \mathcal{S}$ such that for each $\xi \in S$ there exists $F_{\xi} \in \mathcal{F}$ with $\left|f_{\lambda}\left(x_{\xi}\right)-f_{\lambda}\left(z_{\xi}\right)\right| \leq \sigma_{p}$ whenever $\lambda \in F_{\xi}$. 
Let $X$ be any Hausdorff topological space and $x \in X$. A net $f_{\lambda}: X \rightarrow R, \lambda \in \Lambda$, is said to be $(\mathcal{S}, \mathcal{F})$-exhaustive at $x \in X$ iff there exists an $(O)$-sequence $\left(\sigma_{p}\right)_{p}$ in $R$ such that, for every net $x_{\xi}, \xi \in \Xi, \mathcal{S}$-convergent to $x$ and for any $p \in \mathbb{N}$ there are $S \in \mathcal{S}, F \in \mathcal{F}$ with $\left|f_{\lambda}\left(x_{\xi}\right)-f_{\lambda}(x)\right| \leq \sigma_{p}$ for every $\xi \in S$ and $\lambda \in F$.

The net $f_{\lambda}: X \rightarrow R, \lambda \in \Lambda$, is weakly $(\mathcal{S}, \mathcal{F})$-exhaustive at $x \in X$ iff there exists an $(O)$-sequence $\left(\sigma_{p}\right)_{p}$ in $R$ such that, for each net $x_{\xi}, \xi \in \Xi, \mathcal{S}$-convergent to $x$ and for every $p \in \mathbb{N}$ there is a set $S \in \mathcal{S}$ such that for each $\xi \in S$ there exists $F_{\xi} \in \mathcal{F}$ with $\left|f_{\lambda}\left(x_{\xi}\right)-f_{\lambda}(x)\right| \leq \sigma_{p}$ whenever $\lambda \in F_{\xi}$.

Let $\emptyset \neq B \subset X$. We say that a net $f_{\lambda}: X \rightarrow R, \lambda \in \Lambda,(\mathcal{F})$-uniformly converges on $B$ to $f: X \rightarrow R$ iff there exists an $(O)$-sequence $\left(\sigma_{p}\right)_{p}$ such that for every $p \in \mathbb{N}$ there is $F \in \mathcal{F}$ with $\left|f_{\lambda}(x)-f(x)\right| \leq \sigma_{p}$ for each $x \in B$ and $\lambda \in F$.

Let $\mathcal{F}$ be a fixed $(\mathbb{N})$-free filter of $\mathbb{N}$. We say that a sequence $\left(f_{n}\right)_{n}$ in $R^{X}(\mathcal{F}$ c)-converges (filter continuously converges) to $f \in R^{X}$ at $x \in X$ iff there exists an $(O)$-sequence $\left(\sigma_{p}\right)_{p}$ such that for each sequence $\left(x_{n}\right)_{n}$ in $X$ with $(\mathcal{F}) \lim _{n} x_{n}=x$ we get $(R O \mathcal{F}) \lim _{n} f_{n}\left(x_{n}\right)=f(x)$ with respect to $\left(\sigma_{p}\right)_{p}$.

Let now $\Lambda$ and $\Xi$ be two directed sets, $\mathcal{S}$ and $\mathcal{F}$ be a $(\Xi)$-free filter of $\Xi$ and a $(\Lambda)$-free filter of $\Lambda$, respectively. We say that a net $f_{\lambda}: X \rightarrow R, \lambda \in \Lambda,(\mathcal{S}, \mathcal{F} \alpha)$-converges to $f: X \rightarrow R$ at $x \in X$ iff there exists an $(O)$-sequence $\left(\sigma_{p}\right)_{p}$ in $R$ such that, for every net $x_{\xi}, \xi \in \Xi, \mathcal{S}$-convergent to $x$ and for each $p \in \mathbb{N}$ there are $S \in \mathcal{S}, F \in \mathcal{F}$ with $\left|f_{\lambda}\left(x_{\xi}\right)-f(x)\right| \leq \sigma_{p}$ whenever $\xi \in S$ and $\lambda \in F$.

Let $(X, \mathcal{D})$ be a uniform space and $\emptyset \neq B \subset X$. We say that a sequence $\left(f_{n}\right)_{n}$ in $R^{X}$ strongly $(\mathcal{F} c)$-converges to $f \in R^{X}$ on $B$ iff there exists an $(O)$-sequence $\left(\sigma_{p}\right)_{p}$ such that for each pair of sequences $\left(x_{n}\right)_{n},\left(z_{n}\right)_{n}$ in $X$ satisfying condition $\mathrm{H} 1)$ with respect to $\mathcal{F}$ we get $(O \mathcal{F}) \lim _{n}\left(f_{n}\left(x_{n}\right)-f\left(z_{n}\right)\right)=0$ with respect to $\left(\sigma_{p}\right)_{p}$.

A net $f_{\lambda}: X \rightarrow R, \lambda \in \Lambda$, is said to be strongly $(\mathcal{S}, \mathcal{F} \alpha)$-convergent to $f: X \rightarrow R$ on $B$ iff there exists an $(O)$-sequence $\left(\sigma_{p}\right)_{p}$ in $R$ such that, for every pair of nets $x_{\xi}, z_{\xi}, \xi \in \Xi$, satisfying condition H1) with respect to $\mathcal{S}$ and for each $p \in \mathbb{N}$ there are $S \in \mathcal{S}, F \in \mathcal{F}$ with $\left|f_{\lambda}\left(z_{\xi}\right)-f\left(x_{\xi}\right)\right| \leq \sigma_{p}$ whenever $\xi \in S$ and $\lambda \in F$.

A net $f_{\lambda}: X \rightarrow R, \lambda \in \Lambda$, is said to (strongly) $(\mathcal{F} \alpha$ )-converge to $f: X \rightarrow R$ at $x \in X$ (resp. on $B$ ) iff it is (strongly) $(\mathcal{S}, \mathcal{F} \alpha)$-convergent to $f: X \rightarrow R$ at $x \in X$ (resp. on $B$ ) for every directed set $\Xi=(\Xi, \geq)$ and for each $(\Xi)$-free filter $\mathcal{S}$ of $\Xi$.

\section{The Main Results}

We begin with a characterization of (strong) $(\mathcal{S}, \mathcal{F} \alpha)$-convergence of function nets in terms of (strong) $(\mathcal{S}, \mathcal{F})$-exhaustiveness.

Theorem 3.1. Let $(X, \mathcal{D})$ be any uniform space, $\emptyset \neq B \subset X, \Xi=(\Xi, \geq)$ and $\Lambda=(\Lambda, \geq)$ be two directed sets, $\mathcal{S}$ and $\mathcal{F}$ be $a(\Xi)$-free and $(\Lambda)$-free filter of $\Xi$ and $\Lambda$, respectively, and $f_{\lambda}: X \rightarrow R, \lambda \in \Lambda$, be a net of functions, $(\mathcal{F})$-uniformly convergent to $f: X \rightarrow R$ on $B$. Then $\left(f_{\lambda}\right)_{\lambda}$ is strongly $(\mathcal{S}, \mathcal{F} \alpha)$-convergent to $f$ on $B$ if and only if $\left(f_{\lambda}\right)_{\lambda}$ is strongly $(\mathcal{S}, \mathcal{F})$-exhaustive on $B$.

Proof: We begin with the "if" part. Let $\left(\sigma_{p}\right)_{p}$ be an $(O)$-sequence in connection with strong $(\mathcal{S}, \mathcal{F})$-exhaustiveness on $B$ and $x_{\xi}, z_{\xi}, \xi \in \Xi$ be two nets in $X$, satisfying condition H1) with respect to $\mathcal{S}$. Let $\left(\sigma_{p}^{*}\right)_{p}$ be an $(O)$-sequence, associated with $(\mathcal{F})$-uniform convergence of the net $\left(f_{\lambda}\right)_{\lambda}$ to $f$ on $B$. Put $\tau_{p}:=\sigma_{p}+\sigma_{p}^{*}, p \in \mathbb{N}$. We claim that the $(O)$-sequence $\left(\tau_{p}\right)_{p}$ satisfies the condition of strong $(\mathcal{S}, \mathcal{F} \alpha)$-convergence.

Pick arbitrarily $p \in \mathbb{N}$. There are two sets $S \in \mathcal{S}, F \in \mathcal{F}$, with

$$
\left|f_{\lambda}\left(z_{\xi}\right)-f_{\lambda}\left(x_{\xi}\right)\right| \leq \sigma_{p} \quad \text { for all } \xi \in S \text { and } \lambda \in F
$$

By $(\mathcal{F})$-uniform convergence of $\left(f_{\lambda}\right)_{\lambda}$ to $f$ on $B$, there is $F^{*} \in \mathcal{F}$ with

$$
\left|f_{\lambda}\left(x_{\xi}\right)-f\left(x_{\xi}\right)\right| \leq \sigma_{p}^{*} \quad \text { for all } \xi \in \mathcal{S} \text { and } \lambda \in F^{*} .
$$

From (2) and (3) it follows that for each $\lambda \in F \cap F^{*} \in \mathcal{F}$ and $\xi \in S$ we get

$$
\left|f_{\lambda}\left(z_{\xi}\right)-f\left(x_{\xi}\right)\right| \leq\left|f_{\lambda}\left(z_{\xi}\right)-f_{\lambda}\left(x_{\xi}\right)\right|+\left|f_{\lambda}\left(x_{\xi}\right)-f\left(x_{\xi}\right)\right| \leq \sigma_{p}+\sigma_{p}^{*}=\tau_{p},
$$

that is the claim.

We now turn to the "only if" part. Let $\left(\tau_{p}\right)_{p}$ be an $(O)$-sequence, associated with strong $(\mathcal{S}, \mathcal{F} \alpha)$-convergence and $x_{\xi}, z_{\xi}, \xi \in \Xi$ be two nets satisfying H1) with respect to $\mathcal{S}$. Let $\left(\sigma_{p}^{*}\right)_{p}$ be an $(O)$-sequence related to $(\mathcal{F})$-uniform 
convergence on $B$, and put $\sigma_{p}=\tau_{p}+\sigma_{p}^{*}, p \in \mathbb{N}$. We prove that the $(O)$-sequence $\left(\sigma_{p}\right)_{p}$ fulfils the condition of strong $(\mathcal{S}, \mathcal{F})$-exhaustiveness of $\left(f_{\lambda}\right)_{\lambda}$.

Take arbitrarily $p \in \mathbb{N}$. There are two sets $S_{0} \in \mathcal{S}, F_{0} \in \mathcal{F}$, with

$$
\left|f_{\lambda}\left(z_{\xi}\right)-f\left(x_{\xi}\right)\right| \leq \tau_{p} \quad \text { for each } \xi \in S_{0} \text { and } \lambda \in F_{0} .
$$

Let $F^{*}$ be as in (3). From (3) and (4) we obtain

$$
\left|f_{\lambda}\left(z_{\xi}\right)-f_{\lambda}\left(x_{\xi}\right)\right| \leq\left|f_{\lambda}\left(z_{\xi}\right)-f\left(x_{\xi}\right)\right|+\left|f_{\lambda}\left(x_{\xi}\right)-f\left(x_{\xi}\right)\right| \leq \tau_{p}+\sigma_{p}^{*}=\sigma_{p}
$$

whenever $\lambda \in F_{0} \cap F^{*} \in \mathcal{F}$ and $\xi \in S_{0}$. This ends the proof.

By proceeding similarly as in Theorem 3.1, it is possible to prove the following

Theorem 3.2. Let $X$ be a Hausdorff topological space, $x \in X, \Xi, \Lambda, \mathcal{S}$ and $\mathcal{F}$ be as in Theorem 3.1, and $f_{\lambda}: X \rightarrow R$, $\lambda \in \Lambda$, be a net of functions, $(R O \mathcal{F})$-convergent to $f: X \rightarrow R$. Then $\left(f_{\lambda}\right)_{\lambda}$ is $(\mathcal{S}, \mathcal{F} \alpha)$-convergent to $f$ at $x$ if and only if $\left(f_{\lambda}\right)_{\lambda}$ is $(\mathcal{S}, \mathcal{F})$-exhaustive at $x$.

We now relate (strong) weak $(\mathcal{S}, \mathcal{F})$-exhaustiveness with (strong uniform) continuity of the limit function.

Theorem 3.3. Let $X, R, \Xi, \Lambda, \mathcal{S}, \mathcal{F}$ be as in Theorem 3.1, $\emptyset \neq B \subset X$ be fixed, and $f_{\lambda}: X \rightarrow R, \lambda \in \Lambda$, be a function net, $(R O \mathcal{F})$-convergent to $f \in R^{X}$.

Then $\left(f_{\lambda}\right)_{\lambda}$ is strongly weakly $(\mathcal{S}, \mathcal{F})$-exhaustive on $B$ if and only if $f$ is strongly $(\mathcal{S}, \mathcal{S})$-uniformly continuous on $B$.

Proof: We begin with the "only if" part. Let $\left(\sigma_{p}\right)_{p}$ be an $(O)$-sequence associated with strong weak $(\mathcal{S}, \mathcal{F})$-exhaustiveness of $\left(f_{\lambda}\right)_{\lambda}$ on $B$, choose arbitrarily two nets $x_{\xi}, z_{\xi}, \xi \in \Xi$, satisfying condition H1) with respect to $\mathcal{S}$ and a positive integer $p$, and let $S \in \mathcal{S}$ be related to strong weak $(\mathcal{S}, \mathcal{F})$-exhaustiveness. Pick arbitrarily $\xi \in S$, let $F_{\xi}$ be according to strong weak $(\mathcal{S}, \mathcal{F})$-exhaustiveness, and take $\lambda \in F_{\xi}$. By $\left(R O \mathcal{F}\right.$ )-convergence of $\left(f_{\lambda}\right)_{\lambda}$ to $f$ (with respect to a suitable $(O)$-sequence $\left.\left(\sigma_{p}^{*}\right)_{p}\right)$, there is a set $F_{\xi}^{*} \in \mathcal{F}$ with

$$
\left|f_{\lambda}\left(x_{\xi}\right)-f\left(x_{\xi}\right)\right| \vee\left|f_{\lambda}\left(z_{\xi}\right)-f\left(z_{\xi}\right)\right| \leq \sigma_{p}^{*}
$$

for every $\lambda \in F_{\xi}^{*}$. From (5) and strong weak $(\mathcal{S}, \mathcal{F})$-exhaustiveness of $\left(f_{\lambda}\right)_{\lambda}$ we obtain

$$
\left|f\left(x_{\xi}\right)-f\left(z_{\xi}\right)\right| \leq\left|f_{\lambda}\left(x_{\xi}\right)-f\left(x_{\xi}\right)\right|+\left|f_{\lambda}\left(x_{\xi}\right)-f_{\lambda}\left(z_{\xi}\right)\right|+\left|f_{\lambda}\left(z_{\xi}\right)-f\left(z_{\xi}\right)\right| \leq \sigma_{p}+2 \sigma_{p}^{*}
$$

for every $\lambda \in F_{\xi} \cap F_{\xi}^{*} \in \mathcal{F}$ and $\xi \in S$, that is strong $(\mathcal{S}, \mathcal{S})$-uniform continuity of the limit function $f$ on $B$.

We now turn to the "if" part. If $f$ is strongly $(\mathcal{S}, \mathcal{S})$-uniformly continuous on $B$, then there is an $(O)$-sequence $\left(\tau_{p}\right)_{p}$ such that for any $p \in \mathbb{N}$ and for each pair of nets $x_{\xi}, z_{\xi}, \xi \in \Xi$, fulfilling condition H1) with respect to $\mathcal{S}$ there exists a set $S \in \mathcal{S}$ with

$$
\left|f\left(z_{\xi}\right)-f\left(x_{\xi}\right)\right| \leq \tau_{p} \quad \text { for every } \xi \in S .
$$

Pick arbitrarily $\xi \in S$. By $\left(R O \mathcal{F}\right.$ )-convergence of the net $\left(f_{\lambda}\right)_{\lambda}$ to $f$ (with respect to $\left.\left(\sigma_{p}^{*}\right)_{p}\right)$ there exists a set $F_{\xi}^{\prime} \in \mathcal{F}$ such that

$$
\left|f_{\lambda}\left(x_{\xi}\right)-f\left(x_{\xi}\right)\right| \vee\left|f_{\lambda}\left(z_{\xi}\right)-f\left(z_{\xi}\right)\right| \leq \sigma_{p}^{*}
$$

whenever $\lambda \in F_{\xi}^{\prime}$. From (6) and (7) we deduce

$$
\left|f_{\lambda}\left(x_{\xi}\right)-f_{\lambda}\left(z_{\xi}\right)\right| \leq\left|f_{\lambda}\left(x_{\xi}\right)-f\left(x_{\xi}\right)\right|+\left|f\left(x_{\xi}\right)-f\left(z_{\xi}\right)\right|+\left|f_{\lambda}\left(z_{\xi}\right)-f\left(z_{\xi}\right)\right| \leq \tau_{p}+2 \sigma_{p}^{*}
$$

for each $\lambda \in F_{\xi}^{\prime}$, getting strong weak $(\mathcal{S}, \mathcal{F})$-exhaustiveness of the net $\left(f_{\lambda}\right)_{\lambda}$. This ends the proof.

Analogously as Theorem 3.3 it is possible to prove the following

Theorem 3.4. Let $X, R, \Xi, \Lambda, \mathcal{S}, \mathcal{F}$ be as in Theorem 3.2, $x \in X$ be fixed, and $f_{\lambda}: X \rightarrow R, \lambda \in \Lambda$, be a net, $(R O \mathcal{F})$-convergent to $f \in R^{X}$.

Then $\left(f_{\lambda}\right)_{\lambda}$ is weakly $(\mathcal{S}, \mathcal{F})$-exhaustive at $x$ if and only if $f$ is $(\mathcal{S}, \mathcal{S})$-continuous at $x$. 
We now show some differences between filter $(\alpha)$-convergence and strong filter $(\alpha)$-convergence, and between strong filter exhaustiveness and strong weak filter exhaustiveness.

Examples 3.5. (a) In general, $(\mathcal{S}, \mathcal{F} \alpha)$-convergence is strictly weaker than strong $(\mathcal{S}, \mathcal{F} \alpha)$-convergence. Indeed, let $\Xi=(\Xi, \geq)$ be any directed set, $\Lambda=\mathbb{N}$ be with the usual order, $X=[0,1]$ be endowed with the usual metric, $R=\mathbb{R}, f_{n}(x)=x^{n}$ for each $n \in \mathbb{N}$ and $x \in[0,1], f(1)=1$ and $f(x)=0$ for every $x \in[0,1)$. We prove that for every ( $\Xi$ )-free filter $\mathcal{S}$ of $\Xi$ and for each $(\mathbb{N})$-free filter $\mathcal{F}$ of $\mathbb{N}$ the sequence $\left(f_{n}\right)_{n}(\mathcal{S}, \mathcal{F} \alpha)$-converges to $f$, but does not strongly $(\mathcal{S}, \mathcal{F} \alpha)$-converge to $f$ on $B:=[0,1)$. Indeed, if $x_{\xi}, \xi \in \Xi$, is any net in $B, \mathcal{S}$-convergent to $x_{0} \in B$, then $f_{n}\left(x_{\xi}\right)=x_{\xi}^{n}$ and $f\left(x_{0}\right)=0$, and there exist $S \in \mathcal{S}$ and $x_{0}<y<1$ with $x_{\xi}<y$ whenever $\xi \in S$. Choose arbitrarily $\varepsilon>0$. Since $0<y<1$, there exists $n^{\prime} \in \mathbb{N}$ with $y^{n}<\varepsilon$ for each $n \geq n^{\prime}$. Hence for such $n$ 's and $\xi \in S$ we get $0<x_{\xi}^{n}<y^{n}<\varepsilon$. Thus $\left(f_{n}\right)_{n}(\mathcal{S}, \mathcal{F} \alpha)$-converges to $f$ on $B$. On the other hand, pick any pair of nets $x_{\xi}, z_{\xi}, \xi \in \Xi$, in $B$, with $(\mathcal{S}) \lim _{\xi} x_{\xi}=(\mathcal{S}) \lim _{\xi} z_{\xi}=1$. Then we get $f\left(x_{\xi}\right)=0$ for each $\xi \in \Xi$. Now we claim that

$$
\text { for every } S \in \mathcal{S} \text { and } F \in \mathcal{F} \text { there are } \xi^{\prime} \in S, n^{\prime} \in F \text { with } z_{\xi^{\prime}}^{n^{\prime}}>\frac{1}{3} \text {. }
$$

Choose arbitrarily $S \in \mathcal{S}$ and $F \in \mathcal{F}$. As $\lim _{n}\left(1-\frac{1}{n}\right)^{n}=\frac{1}{e}$, there is $n_{0} \in \mathbb{N}$ with

$$
\left(1-\frac{1}{n}\right)^{n}>\frac{1}{3} \quad \text { for every } n \geq n_{0} .
$$

Let $n^{\prime}$ be any integer greater than $n_{0}$ and belonging to $F$. Since $(\mathcal{S}) \lim _{\xi} z_{\xi}=1$, in correspondence with $n^{\prime}$ there is $S_{n^{\prime}} \in \mathcal{S}$ with $z_{\xi}>1-\frac{1}{n^{\prime}}$ whenever $\xi \in S_{n^{\prime}}$. Let $\xi^{\prime} \in S \cap S_{n^{\prime}}$. Since $z_{\xi^{\prime}}>1-\frac{1}{n^{\prime}}$, taking into account (9) we get

$$
z_{\xi^{\prime}}^{n^{\prime}}>\left(1-\frac{1}{n^{\prime}}\right)^{n^{\prime}}>\frac{1}{3}
$$

that is (8). Thus, the sequence $\left(f_{n}\right)_{n}$ does not strongly $(\mathcal{S}, \mathcal{F} \alpha)$-converge to $f$ on $B$.

(b) We now see that, in general, strong weak $(\mathcal{S}, \mathcal{F})$-exhaustiveness is strictly weaker than strong $(\mathcal{S}, \mathcal{F})$-exhaustiveness. Let $\Xi=(\Xi, \geq)$ be an abstract directed set, $\Lambda=(\Lambda, \geq)=] 1,+\infty[$ be directed by the usual order, $X=\mathbb{R}$ be endowed with the usual distance, $B=[-1,1], v$ be the Lebesgue measure on $\mathbb{R}$, and $R$ be the space of all bounded $v$-measurable real-valued functions defined on $\mathbb{R}$, with identification up to $v$-null sets, where order convergence is equal to almost convergence dominated by an element of $R$, and thus it does not have a topological nature (see also Boccuto and Dimitriou (2015)). For every real number $a$, let $\underline{a}$ be that function which associates to every element $x \in X$ the constant $a$. Let $\mathcal{S}, \mathcal{F}$ be any fixed $(\Xi)$-free filter of $\Xi$ and any fixed $(\Lambda)$-free filter of $\Lambda$, respectively, and let us define $f_{\lambda}: X \rightarrow R, \lambda \in \Lambda$, by

$$
f_{\lambda}(x)= \begin{cases}\underline{0}, & \text { if } x \in]-\infty,-1 / \lambda] \cup\{0\} \cup[1 / \lambda,+\infty[, \\ \underline{\lambda}, & \text { otherwise. }\end{cases}
$$

It is not difficult to see that the net $\left(f_{\lambda}\right)_{\lambda}(R O \mathcal{F})$-converges to 0 with respect to the $(O)$-sequence $\sigma_{p}:=\frac{1}{p}, p \in \mathbb{N}$, and so from Theorem 3.3 it follows that $\left(f_{\lambda}\right)_{\lambda}$ is strongly weakly $(\mathcal{S}, \mathcal{F})$-exhaustive at every point $x \in \mathbb{R}$. We now prove that $\left(f_{\lambda}\right)_{\lambda}$ is not strongly $(\mathcal{S}, \mathcal{F})$-exhaustive at 0 . First of all observe that, if $\left(\sigma_{p}\right)_{p}$ is any $(O)$-sequence in $R$, then there is a positive real number $M^{\prime}$ with $\sigma_{p}(x) \leq \sigma_{1}(x) \leq M^{\prime}$ for each $p \in \mathbb{N}$ and $x \in X$. Set $z_{\xi}=0$ for each $\xi \in \Xi$, and let $x_{\xi}, \xi \in \Xi$, be any sequence of elements of [-1,1], with $\lim _{\xi \in \Xi} x_{\xi}=0$ in the usual sense. This implies that $(\mathcal{S}) \lim _{\xi \in \Xi} x_{\xi}=0$, and hence $x_{\xi}, z_{\xi}, \xi \in \Xi$, satisfy condition H1) with respect to $\mathcal{S}$. Choose arbitrarily $S \in \mathcal{S}$. We claim that

$$
\text { for every } \xi \in \Xi \text { there is } \xi^{\prime} \in S \text { with } \xi^{\prime} \geq \xi \text {. }
$$

Indeed, if (10) is not true, then there exists $\xi_{*} \in \Xi$ with $\xi \nsupseteq \xi_{*}$ whenever $\xi \in S$. Thus, since $\mathcal{S}$ is a ( $\Xi$ )-free filter of $\Xi$, then the set $M_{\xi_{*}}:=\left\{\xi \in \Xi: \xi \geq \xi_{*}\right\}$ belongs to $\mathcal{S}$, and hence $\emptyset=S \cap M_{\xi_{*}} \in \mathcal{S}$ : this is absurd. So the claim is 
proved. Analogously to (10), it is possible to prove that for every ( $\Lambda$ )-free filter $\mathcal{F}$ of $\Lambda$ and for each $F \in \mathcal{F}$ there is $\lambda^{\prime} \in F$ with $\lambda^{\prime}>M^{\prime}$. Since $\lim _{\xi \in \Xi} x_{\xi}=0$ in the usual sense, by virtue of (10) there exists $\xi^{\prime} \in S$ with $\left|x_{\xi^{\prime}}\right| \leq 1 / \lambda^{\prime}$.

Hence we get

$$
\left|f_{\lambda^{\prime}}\left(x_{\xi^{\prime}}\right)-f_{\lambda^{\prime}}\left(z_{\xi^{\prime}}\right)\right|=\left|f_{\lambda^{\prime}}\left(x_{\xi^{\prime}}\right)-f_{\lambda^{\prime}}(0)\right|=\left|f_{\lambda^{\prime}}\left(x_{\xi^{\prime}}\right)\right|=\underline{\lambda^{\prime}}>\underline{M^{\prime}},
$$

and thus

$$
\left|f_{\lambda}^{\prime}\left(x_{\xi^{\prime}}\right)-f_{\lambda}^{\prime}\left(z_{\xi^{\prime}}\right)\right| \not \sigma_{1} \text {. }
$$

This proves that the net $\left(f_{\lambda}\right)_{\lambda}$ is not strongly $(\mathcal{S}, \mathcal{F})$-exhaustive at 0 .

We now give a characterization of (strong uniform) continuity of lattice group-valued functions with respect to a pair of filters, extending Baláž, Červeňanskij, Kostyrko and Šalát (2002), Theorem 3 and Boccuto, Dimitriou, Papanastassiou and Wilczyński (2014), Theorem 4.6.

Theorem 3.6. Let $(X, \mathcal{D})$ be a uniform space, $(\Xi, \geq)$ be a directed set, $f: X \rightarrow R, \emptyset \neq B \subset X, \mathcal{S}_{1}$ and $\mathcal{S}_{2}$ be any two fixed $(\Xi)$-free filters of $\Xi$. Suppose that for every point $x \in X$ there is a net $y_{\xi}, \xi \in \Xi$, in $X$ with $\left(\mathcal{S}_{1}\right) \lim _{\xi} y_{\xi}=x$. Then the following results hold.

(a) If $\mathcal{S}_{1} \backslash \mathcal{S}_{2} \neq \emptyset$, then $f$ is strongly $\left(\mathcal{S}_{1}, \mathcal{S}_{2}\right)$-uniformly continuous on $B$ if and only if $f$ is constant.

(b) If $\mathcal{S}_{1} \subset \mathcal{S}_{2}$ and $D_{\xi}, \xi \in \Xi$, is a decreasing net in $\mathcal{D}$ with the property that for every $U \in \mathcal{D}$ there is $\xi \in \Xi$ with $D_{\xi} \subset U$, then $f$ is strongly $\left(\mathcal{S}_{1}, \mathcal{S}_{2}\right)$-uniformly continuous on $B$ if and only if $f$ is strongly uniformly continuous on B.

Proof: (a) The "if" part is straightforward.

Concerning the "only if" part, pick arbitrarily $x, y \in R$ with $x \neq y$, and let us show that $f(x)=f(y)$. Otherwise, for every $(O)$-sequence $\left(\sigma_{p}\right)_{p}$ in $R$ there is $\bar{p} \in \mathbb{N}$ with

$$
|f(x)-f(y)| \not \leq \sigma_{\bar{p}}
$$

(see also Boccuto, Dimitriou, Papanastassiou and Wilczyński (2014)). By hypothesis, there is a set $C \in \mathcal{S}_{1} \backslash \mathcal{S}_{2}$. Note that $C \neq \emptyset$ and $C \neq \Xi$. Let $\left(y_{\xi}\right)_{\xi}$ be a sequence, with $\left(\mathcal{S}_{1}\right) \lim _{\xi} y_{\xi}=x$. Put $z_{\xi}=y_{\xi}$ for $\xi \in C, z_{\xi}=y$ for $\xi \in \Xi \backslash C, x_{\xi}=x$ for each $\xi \in \Xi$. It is not difficult to check that $x_{\xi}, z_{\xi}, \xi \in \Xi$, fulfil condition H1) with respect to $\mathcal{S}_{1}$. Thanks to strong $\left(\mathcal{S}_{1}, \mathcal{S}_{2}\right)$-uniform continuity, also condition H2) (with respect to $\left.\mathcal{S}_{2}\right)$ is satisfied, that is

$$
\left\{\xi \in \Xi:\left|f\left(x_{\xi}\right)-f\left(z_{\xi}\right)\right| \leq \sigma_{p}\right\} \in \mathcal{S}_{2} \quad \text { for every } p \in \mathbb{N} \text {. }
$$

But $E_{\bar{p}}:=\left\{\xi \in \Xi:\left|f\left(x_{\xi}\right)-f\left(z_{\xi}\right)\right| \leq \sigma_{\bar{p}}\right\} \subset C$, and hence $E_{\bar{p}} \notin \mathcal{S}_{2}$, which contradicts (12). This proves (a).

We now turn to (b). We begin with the "if" part. Let $x_{\xi}, x_{\xi}, \xi \in \Xi$, be two nets, satisfying condition H1) with respect to $\mathcal{S}_{1}$. This means that for every $D \in \mathcal{D},\left\{\xi \in \Xi:\left(x_{\xi}, z_{\xi}\right) \in D\right\} \in \mathcal{S}_{1}$. Let $\left(\sigma_{p}\right)_{p}$ be an $(O)$-sequence in $R$, associated with strong uniform continuity of $f$ on $B$. Fix now arbitrarily $p \in \mathbb{N}$ and choose $D \in \mathcal{D}$, in correspondence with strong uniform continuity. By hypothesis, we have

$$
\left\{\xi \in \Xi:\left(x_{\xi}, z_{\xi}\right) \in D\right\} \subset\left\{\xi \in \Xi:\left|f\left(x_{\xi}\right)-f\left(z_{\xi}\right)\right| \leq \sigma_{p}\right\} \in \mathcal{S}_{1} \subset \mathcal{S}_{2},
$$

obtaining the assertion.

We now turn to the "only if" part. Suppose that $f$ is strongly $\left(\mathcal{S}_{1}, \mathcal{S}_{2}\right)$-uniformly continuous on $B$, but not strongly uniformly continuous on $B$. By hypothesis there is $\bar{p} \in \mathbb{N}$ such that for every $\xi \in \Xi$ there are $x_{\xi}, z_{\xi} \in B$ with $\left(x_{\xi}, z_{\xi}\right) \in D_{\xi}$ and $\left|f\left(x_{\xi}\right)-f\left(z_{\xi}\right)\right| \not \leq \sigma_{\bar{p}}$. The nets $x_{\xi}, z_{\xi}, \xi \in \Xi$, fulfil H1) with respect to $\mathcal{S}_{1}$ and H2) with respect to $\mathcal{S}_{2}$, by strong $\left(\mathcal{S}_{1}, \mathcal{S}_{2}\right)$-uniform continuity of $f$. But this contradicts the fact that $\left\{\xi \in \Xi:\left|f\left(x_{\xi}\right)-f\left(z_{\xi}\right)\right| \leq \sigma_{\bar{p}}\right\}=\emptyset \notin \mathcal{S}_{2}$. This completes the proof.

By an analogous argument as in Theorem 3.6 it is possible to prove the following 
Theorem 3.7. Let $X$ be a Hausdorff topological space, $f: X \rightarrow R,(\Xi, \geq)$ be a directed set, $\mathcal{S}_{1}$ and $\mathcal{S}_{2}$ be two fixed ( $\Xi)$-free filters of $\Xi, x \in X$ be such that there is a net $y_{\xi}, \xi \in \Xi$, in $X$ with $\left(\mathcal{S}_{1}\right) \lim _{\xi} y_{\xi}=x$.

Then the following results hold.

(a) If $\mathcal{S}_{1} \backslash \mathcal{S}_{2} \neq \emptyset$, then $f$ is $\left(\mathcal{S}_{1}, \mathcal{S}_{2}\right)$-continuous at $x$ if and only if $f$ is constant.

(b) If $\mathcal{S}_{1} \subset \mathcal{S}_{2},\left(\mathcal{T}_{x}, \subset\right)$ is the set of all neighborhoods of $x$ and $D_{\xi}, \xi \in \Xi$, is a decreasing net in $\mathcal{T}_{x}$, such that for every $U \in \mathcal{D}$ there is $\xi \in \Xi$ with $D_{\xi} \subset U$, then $f$ is $\left(\mathcal{S}_{1}, \mathcal{S}_{2}\right)$-continuous at $x$ if and only if it is continuous at $x$.

Now we compare filter $(\alpha)$-convergence with respect to a pair of filters with usual filter continuous convergence with respect to a single filter. The following result extends Gregoriades and Papanastassiou (2008), Proposition 3.2.2, and we use the tool of transfinite induction, similarly to Boccuto, Dimitriou, Papanastassiou and Wilczyński (2011), Theorem 3.3.

Theorem 3.8. Let $(X, \mathcal{D})$ be a uniform space, $\left(U_{k}\right)_{k}$ be a decreasing base of elements of $\mathcal{D}, \emptyset \neq B \subset X, \mathcal{F}$ be an $(\mathbb{N})$-free filter of $\mathbb{N}$, satisfying condition (1) and $f_{n}: X \rightarrow R, n \in \mathbb{N}$, be a function sequence, strongly $(\mathcal{F} \alpha)$-convergent to $f \in R^{X}$ on $B$. Then $\left(f_{n}\right)_{n}$ is strongly $(\mathcal{F} c)$-convergent to $f$ on $B$.

Conversely, if $\mathcal{F}$ satisfies condition (1) and $f_{n}: X \rightarrow R, n \in \mathbb{N}$, strongly $(\mathcal{F} c)$-converges to $f$ on $B$, then $\left(f_{n}\right)_{n}$ strongly $(\mathcal{F} \alpha)$-converges to $f$ on $B$.

Proof: The first part is straightforward.

We now turn to the last part. Fix a directed set $\Xi=(\Xi, \geq)$ and a $(\Xi)$-free filter $\mathcal{S}$ of $\Xi$. Let $\mathcal{J}$ and $\mathcal{I}$ be the dual ideals of $\mathcal{S}$ and $\mathcal{F}$, respectively, and $\left(\sigma_{p}\right)_{p}$ be an $(O)$-sequence associated with strong $(\mathcal{F} c)$-convergence of $\left(f_{n}\right)_{n}$ to $f$. Take any two nets $x_{\xi}, z_{\xi}, \xi \in \Xi$, satisfying condition H1) with respect to $\mathcal{S}$. We claim that $\left(\sigma_{p}\right)_{p}$ is the required $(O)$-sequence for strong $(\mathcal{S}, \mathcal{F} \alpha)$-convergence of $\left(f_{n}\right)_{n}$ to $f$.

If it is not the case, then there exists $p \in \mathbb{N}$ such that for any $A \in \mathcal{I}$ and $E \in \mathcal{J}$ there are $n \in \mathbb{N} \backslash A$ and $\xi \in \Xi \backslash E$, with

$$
\left|f_{n}\left(z_{\xi}\right)-f\left(x_{\xi}\right)\right| \not \leq \sigma_{p} .
$$

Fix $k \in \mathbb{N}$. By H1) there is a set $E^{k} \in \mathcal{J}$ with $\left(x_{\xi}, z_{\xi}\right) \in U_{k}$ whenever $\xi \notin E^{k}$. Let us consider $A_{0}^{k}=\emptyset$ : so there are $n_{0}^{k} \in \mathbb{N}$ and $\xi \in \Xi \backslash E^{k}$ with $\left|f_{n_{0}^{k}}\left(z_{\xi_{0}}\right)-f\left(x_{\xi_{0}}\right)\right| \not \leq \sigma_{p}$.

We now proceed by transfinite induction. Assume to have chosen $n_{\beta}^{k}$ and $\xi_{\beta}^{k}$ for $\beta \leq \alpha$, where $\alpha$ is an ordinal of first kind, and that $A_{\alpha}^{k}=\left\{n_{\beta}^{k}: \beta \leq \alpha\right\} \in \mathcal{I}$. There exist $n_{\alpha+1}^{k} \in \mathbb{N} \backslash A_{\alpha}^{k}$ and $\xi_{\alpha+1}^{k} \in \Xi \backslash E^{k}$ with $\left(x_{\xi_{\alpha+1}^{k}}, z_{\xi_{\alpha+1}^{k}}\right) \in U_{k}$ and $\left|f_{n_{\alpha+1}^{k}}\left(z_{\xi_{\alpha+1}^{k}}\right)-f\left(x_{\xi_{\alpha+1}^{k}}\right)\right| \not \leq \sigma_{p}$. In this case, set $A_{\alpha+1}^{k}=A_{\alpha}^{k} \cup\left\{n_{\alpha+1}^{k}\right\}$. When $\alpha$ is an ordinal of second kind, if we have chosen $n_{\beta}^{k}$ and $\xi_{\beta}^{k}$ for $\beta<\alpha$, set $A_{\alpha}^{k}=\bigcup_{\beta<\alpha} A_{\beta}^{k}$. This procedure ends at some countable limit ordinal $\alpha_{k}$, since otherwise we would obtain a strictly increasing sequence $\left\{A_{\alpha}^{k}: \alpha<\omega_{1}\right\}$ of subsets of $\mathbb{N}$. Hence, $A_{\alpha_{k}}^{k} \notin \mathcal{I}$. Put $P_{k}:=A_{\alpha_{k}}^{k}$ for every $k \in \mathbb{N}$. By virtue of Remark 2.1 (b) there is a disjoint sequence $\left(B_{k}\right)_{k}$ in $\mathcal{I}$ with $B_{k} \subset P_{k}$ for all $k \in \mathbb{N}$ and $\bigcup_{k=1}^{\infty} B_{k} \notin \mathcal{I}$. Let $\bar{x}$ be any fixed element of $B$, and for each $n \in \mathbb{N}$ set $v_{n}=w_{n}=\bar{x}$ if $n \notin \bigcup_{k=1}^{\infty} B_{k}$ and $v_{n}=x_{\xi_{\beta}^{k}}, w_{n}=z_{\xi_{\beta}^{k}}$, if $n \in B_{k}, n=n_{\beta}^{k}$ (note that the integer $k$ is uniquely determined, since the $B_{k}$ 's are disjoint, and thus the $v_{n}$ 's and the $w_{n}$ 's are well-defined).

The sequences $\left(v_{n}\right)_{n},\left(w_{n}\right)_{n}$ satisfy property $\left.\mathrm{H} 1\right)$ with respect to $\mathcal{F}$. Indeed, it is not difficult to see that

$$
\left\{n \in \mathbb{N}:\left(v_{n}, w_{n}\right) \notin U_{k}\right\} \subset \bigcup_{j=1}^{k-1} B_{j}
$$

and $\bigcup_{j=1}^{k-1} B_{j} \in \mathcal{I}$ for every $k \in \mathbb{N}$. Moreover we get

$$
\left\{n \in \mathbb{N}:\left|f_{n}\left(w_{n}\right)-f\left(v_{n}\right)\right| \not \sigma_{p}\right\}=\bigcup_{k=1}^{\infty} B_{k} \notin \mathcal{I},
$$


obtaining a contradiction with strong $(\mathcal{F} c)$-convergence. This ends the proof.

Analogously as in Theorem 3.8, it is possible to prove the following

Theorem 3.9. Let $X$ be a Hausdorff topological space, $x \in X,\left(U_{k}\right)_{k}$ be a decreasing base of neighborhoods of $x$, $\mathcal{F}$ be an $(\mathbb{N})$-free filter of $\mathbb{N}$, and $f_{n}: X \rightarrow R, n \in \mathbb{N}$, be a sequence, $(\mathcal{F} \alpha)$-convergent to $f \in R^{X}$ at $x$. Then $\left(f_{n}\right)_{n}$ is $(\mathcal{F} c)$-convergent to $f$ at $x$.

Conversely, if $\mathcal{F}$ satisfies condition $(1)$ and $f_{n}: X \rightarrow R, n \in \mathbb{N}$, is $(\mathcal{F} c)$-convergent to $f$ at $x$, then $\left(f_{n}\right)_{n}$ is $(\mathcal{F} \alpha)$-convergent to $f$ at $x$.

Example 3.10. In Theorems 3.8 and 3.9, in general condition (1) cannot be dropped, even when $R=\mathbb{R}$. To this aim, we first construct a partition $\left(P_{k}\right)_{k}$ of $\mathbb{N}$, such that the asymptotic density of $P_{k}$ is $\frac{1}{2^{k}}$ for every $k$. We will define a function $\bar{\phi}: \mathbb{N} \rightarrow \mathbb{N}$ as follows.

At the first step, set $\bar{\phi}(1)=1$ and $\psi_{1}=\{1\}$.

At the second step, let us consider the number 2 and the finite sequence $\psi_{2}=\{1,2,1\}$, and put $\bar{\phi}(2)=2$ and $\bar{\phi}(3)=1$. Note that $\psi_{2}$ is formed by the terms of $\psi_{1}, 2$ and the members of $\psi_{1}$ again.

At the third step, let us take the number 3 and the finite sequence formed by $\psi_{2}, 3$ and $\psi_{2}$ again, namely $\psi_{3}:=$ $\{1,2,1,3,1,2,1\}$, and set $\bar{\phi}(4)=3, \bar{\phi}(5)=\bar{\phi}(7)=1, \bar{\phi}(6)=2$.

Proceeding by induction, at the $k$-th step the sequence $\psi_{k}$ will be formed by the elements of $\psi_{k-1}$, the integer $k$ and again the numbers of $\psi_{k-1}$. In this way, we construct a function $\bar{\phi}$ with $\bar{\phi}\left(2^{k-1}\right)=k$ and $\bar{\phi}\left(n+2^{k-1}\right)=\bar{\phi}(n)$ whenever $n=1, \ldots, 2^{k-1}-1$. Thus, by the induction technique, it is possible to define $\bar{\phi}$ on the whole of $\mathbb{N}$, and we will get $\bar{\phi}(\mathbb{N})=\mathbb{N}$.

Now, for every $k \in \mathbb{N}$, set

$$
P_{k}:=\bar{\phi}^{-1}(\{k\})=\left\{2^{k-1}+n 2^{k}: n \in \mathbb{N} \cup\{0\}\right\}
$$

By construction, we have that $\bigcup_{k=1}^{\infty} P_{k}=\mathbb{N}$, the $P_{k}$ 's are disjoint and the asymptotic density of $P_{k}$ is $2^{-k}$ for every $k$. Moreover, again by construction, we get

$$
\frac{\sharp\left(P_{k} \cap\{1, \ldots, n\}\right)}{n} \leq 2^{-k+1} \quad \text { for all } k, n \in \mathbb{N} .
$$

Let $\left(A_{k}\right)_{k}$ be any sequence of subsets of $\mathbb{N}$ with null asymptotic density and such that $A_{k} \subset P_{k}$ for each $k$. Choose arbitrarily $\varepsilon>0$. There exists $k_{0} \in \mathbb{N}$ with $\frac{1}{2^{k_{0}-1}} \leq \varepsilon$. In correspondence with $\frac{\varepsilon}{2 k_{0}}$, for every $k \in\left[1, k_{0}\right]$ there is $n_{k} \in \mathbb{N}$ with

$$
\frac{\sharp\left(A_{k} \cap\{1, \ldots, n\}\right)}{n} \leq \frac{\varepsilon}{2 k_{0}} \quad \text { whenever } n \geq n_{k} .
$$

Set $n_{0}:=\max \left\{n_{1}, \ldots, n_{k_{0}}\right\}$ and pick arbitrarily $n \geq n_{0}$. We have

$$
\begin{aligned}
& \frac{\sharp\left(\left(\bigcup_{k=1}^{\infty} A_{k}\right) \cap\{1, \ldots, n\}\right)}{n}=\frac{\sum_{k=1}^{k_{0}} \sharp\left(A_{k} \cap\{1, \ldots, n\}\right)}{n} \\
+ & \frac{\sharp\left(\left(\bigcup_{k=k_{0}+1}^{\infty} A_{k}\right) \cap\{1, \ldots, n\}\right)}{n} \leq \frac{\varepsilon}{2}+\frac{\sharp\left(\left(\bigcup_{k=k_{0}+1}^{\infty} P_{k}\right) \cap\{1, \ldots, n\}\right)}{n} \\
= & \frac{\varepsilon}{2}+\sum_{k=k_{0}+1}^{\infty} \frac{\sharp\left(P_{k} \cap\{1, \ldots, n\}\right)}{n} \leq \frac{\varepsilon}{2}+\sum_{k=k_{0}+1}^{\infty} \frac{1}{2^{k-1}}=\frac{\varepsilon}{2}+\frac{1}{2^{k_{0}}} \leq \frac{\varepsilon}{2}+\frac{\varepsilon}{2}=\varepsilon,
\end{aligned}
$$

since the $k$ 's which form the sum $\sum_{k=k_{0}+1}^{\infty} \frac{\sharp\left(P_{k} \cap\{1, \ldots, n\}\right)}{n}$ are at most finite, because there is a $k^{*} \in \mathbb{N}$ with min $P_{k}>$ $n$ for every $k \geq k^{*}$. Thus we obtain

$$
\lim _{n} \frac{\sharp\left(\left(\bigcup_{k=1}^{\infty} A_{k}\right) \cap\{1, \ldots, n\}\right)}{n}=0 .
$$


So we have found a partition $\left(P_{k}\right)_{k}$ of $\mathbb{N}$ with $P_{k} \notin \mathcal{I}_{\text {st }}$ for every $k \in \mathbb{N}$, and such that

$$
\bigcup_{k=1}^{\infty} A_{k} \in \mathcal{I}_{\mathrm{st}}
$$

whenever $\left(A_{k}\right)_{k}$ is a sequence in $\mathcal{I}_{\text {st }}$ with $A_{k} \subset P_{k}$ for all $k \in \mathbb{N}$.

Let now $X=(0,1]$ be endowed with the usual metric, $\mathcal{F}=\mathcal{F}_{\text {st }},\left(P_{k}\right)_{k}$ be as in (14), $f \equiv 0$ and $f_{n}=\chi_{\left(\frac{1}{k+1}, \frac{1}{k}\right]}$ if $n \in P_{k}, n, k \in \mathbb{N}$.

We prove that $\left(f_{n}\right)_{n}\left(\mathcal{F}_{\text {st }} c\right)$-converges to $f$ at 0 . Let $\left(x_{n}\right)_{n}$ be a sequence $\mathcal{F}_{\text {st }}$-convergent to 0 , set

$$
\begin{gathered}
B_{0}:=\left\{n \in \mathbb{N}: x_{n}>1 \text { and } f_{n}\left(x_{n}\right) \neq 0\right\}=\left\{n \in \mathbb{N}: x_{n}>1 \text { and } f_{n}\left(x_{n}\right)=1\right\}, \\
B_{k}:=\left\{n \in \mathbb{N}: \frac{1}{k+1}<x_{n} \leq \frac{1}{k} \text { and } f_{n}\left(x_{n}\right) \neq 0\right\}= \\
\quad=\left\{n \in \mathbb{N}: \frac{1}{k+1}<x_{n} \leq \frac{1}{k} \text { and } f_{n}\left(x_{n}\right)=1\right\}, \quad k \geq 1 .
\end{gathered}
$$

Note that $B_{0} \in \mathcal{I}_{\mathrm{st}}, B_{k} \subset P_{k}$ and $B_{k} \in \mathcal{I}_{\mathrm{st}}, k \in \mathbb{N}$. Thanks to (16), we get

$$
\left\{n \in \mathbb{N}: f_{n}\left(x_{n}\right) \neq 0\right\}=\left\{n \in \mathbb{N}: f_{n}\left(x_{n}\right)=1\right\}=\bigcup_{k=0}^{\infty} B_{k} \in \mathcal{I}_{s t},
$$

and hence $\left(\mathcal{F}_{s t}\right) \lim _{n} f_{n}\left(x_{n}\right)=0$. Thus $\left(f_{n}\right)_{n}$ is $\left(\mathcal{F}_{\text {st }} c\right)$-convergent to $f$ at 0 .

In order to prove that $\left(f_{n}\right)_{n}$ does not $\left(\mathcal{S}, \mathcal{F}_{\text {st }} \alpha\right)$-converge to $f$ at 0 for any directed set $(\Xi, \geq)$ and for any $(\Xi)$-free filter $\mathcal{S}$ of $\Xi$, it is enough to show that for every set $\left\{x_{\xi}: \xi \in \Xi\right\} \subset(0,1]$ and $D \in \mathcal{F}_{\text {st }}$ there are $\xi \in \Xi$ and $n \in D$ with $f_{n}\left(x_{\xi}\right)=1$. Indeed, pick arbitrarily $\xi \in \Xi$ and $D \in \mathcal{F}_{\text {st }}$. There is $k \in \mathbb{N}$ with $x_{\xi} \in\left(\frac{1}{k+1}, \frac{1}{k}\right]$. Let $P_{k}$ be as in (14). As $P_{k} \notin \mathcal{I}_{\text {st }}$, then $P_{k} \cap D \neq \emptyset$. For every $n \in P_{k} \cap D$ we have by definition $f_{n}\left(x_{\xi}\right)=1$, getting that, in Theorem 3.9, in general condition (1) cannot be eliminated.

We now give the following comparison between (strong) $\mathcal{F}$-exhaustiveness and (strong) $(\mathcal{S}, \mathcal{F}$ )-exhaustiveness.

Theorem 3.11. Let $(X, \mathcal{D})$ be any uniform space, $\emptyset \neq B \subset X, \Lambda$ and $\Xi$ be two directed sets, $\mathcal{S}$ and $\mathcal{F}$ be $a(\Xi)$-free filter of $\Xi$ and $a(\Lambda)$-free filter of $\Lambda$, respectively, and $f_{\lambda}: X \rightarrow R, \lambda \in \Lambda$, be a function net, strongly $\mathcal{F}$-exhaustive on $B$. Then $\left(f_{\lambda}\right)_{\lambda}$ is strongly $(\mathcal{S}, \mathcal{F})$-exhaustive on $B$.

Conversely, if $D_{\xi}, \xi \in \Xi$, is a decreasing net in $\mathcal{D}$, such that for each $U \in \mathcal{D}$ there exists $\xi \in \Xi$ with $D_{\xi} \subset U$, and $\left(f_{\lambda}\right)_{\lambda}$ is strongly $(\mathcal{S}, \mathcal{F})$-exhaustive on $B$, then $\left(f_{\lambda}\right)_{\lambda}$ is strongly $\mathcal{F}$-exhaustive on $B$.

Proof: We begin with the first part. Let $\left(\sigma_{p}\right)_{p}$ be an $(O)$-sequence, related to strong $\mathcal{F}$-exhaustiveness, and $x_{\xi}, z_{\xi}$, $\xi \in \Xi$, be any pair of nets, satisfying condition H1) with respect to $\mathcal{S}$. Then for any $D \in \mathcal{D}$ there exists a set $S \in \mathcal{S}$, with

$$
\left(x_{\xi}, z_{\xi}\right) \in D \quad \text { whenever } \xi \in S .
$$

Pick arbitrarily $p \in \mathbb{N}$ and let us associate with $p$ a set $F \in \mathcal{F}$ and an element $D \in \mathcal{D}$ in connection with strong $\mathcal{F}$-exhaustiveness. In correspondence with $D$, let $S \in \mathcal{S}$ be related to (17). For every $\xi \in S$ and $\lambda \in F$ we have $\left|f_{\lambda}\left(x_{\xi}\right)-f_{\lambda}\left(z_{\xi}\right)\right| \leq \sigma_{p}$, getting strong $(\mathcal{S}, \mathcal{F})$-exhaustiveness of $\left(f_{\lambda}\right)_{\lambda}$ on $B$.

We now turn to the last part. Suppose that $\left(f_{\lambda}\right)_{\lambda}$ is strongly $(\mathcal{S}, \mathcal{F})$-exhaustive on $B$, but not strongly $\mathcal{F}$-exhaustive on $B$. Then there is $\bar{p} \in \mathbb{N}$ such that for every $\xi \in \Xi$ there are $x_{\xi}, z_{\xi} \in B$ with $\left(x_{\xi}, z_{\xi}\right) \in D_{\xi}$ and for every $\xi \in \Xi$ and $F \in \mathcal{F}$ there is a $\lambda \in F$ with

$$
\left|f_{\lambda}\left(x_{\xi}\right)-f_{\lambda}\left(z_{\xi}\right)\right| \not \leq \sigma_{\bar{p}} .
$$

Observe that the nets $x_{\xi}, z_{\xi}, \xi \in \Xi$, fulfil condition H1) with respect to $\mathcal{S}$. By strong $(\mathcal{S}, \mathcal{F})$-exhaustiveness of the net $\left(f_{\lambda}\right)_{\lambda}$, there are $S_{0} \in \mathcal{S}$ and $F_{0} \in \mathcal{F}$, with $\left|f_{\lambda}\left(x_{\xi}\right)-f_{\lambda}\left(z_{\xi}\right)\right| \leq \sigma_{\bar{p}}$ for each $\xi \in S_{0}$ and $\lambda \in F_{0}$. This contradicts (18) used with $F=F_{0}$ and ends the proof.

Similarly as Theorem 3.11 it is possible to prove the following 
Theorem 3.12. Let $X$ be a Hausdorff topological space, $x \in X$ be fixed, $\left(\mathcal{T}_{x}, \subset\right)$ be the set of all neighborhoods of $x ; \Lambda, \Xi, \mathcal{S}, \mathcal{F}$ be as in Theorem 3.11, and $f_{\lambda}: X \rightarrow R, \lambda \in \Lambda$, be a net, $\mathcal{F}$-exhaustive at $x$. Then $\left(f_{\lambda}\right)_{\lambda}$ is $(\mathcal{S}, \mathcal{F})$-exhaustive at $x$.

Conversely, if $D_{\xi}, \xi \in \Xi$, is a decreasing net in $\mathcal{T}_{x}$ such that for every $U \in \mathcal{T}_{x}$ there is $\xi \in \Xi$ with $D_{\xi} \subset U$, and $\left(f_{\lambda}\right)_{\lambda}$ is $(\mathcal{S}, \mathcal{F})$-exhaustive at $x$, then $\left(f_{\lambda}\right)_{\lambda}$ is $\mathcal{F}$-exhaustive at $x$.

As an application, we give a result, which extends some classical versions of Ascoli theorems related to exhaustiveness (see also Athanassiadou, Boccuto, Dimitriou and Papanastassiou (2012), Theorem 3.7, Caserta, Di Maio and Kočinac (2011), Theorem 4.7, Gregoriades and Papanastassiou (2008), Theorem 3.1.1) and some other Ascoli-type theorems, where the topological space on which the involved functions are defined is not necessarily metrizable (see for instance Gregoriades and Papanastassiou (2008), Theorems 3.2.19, 3.2.20, and Kelley (1955), Theorems 7.6, 7.18 and 7.21). The approach which we use in investigating the concepts of filter $(\alpha)$-convergence and exhaustiveness with respect to a pair of filters, and consequently (strong uniform) continuity, is very natural to formulate some concepts of "filter closedness" and "filter compactness" in connection with convergences, which are not generated by any Hausdorff topology. Note that in the space $L^{0}([0,1], \Sigma, v)$ of all measurable functions on $[0,1]$ with respect to the $\sigma$-algebra $\Sigma$ of all Borel subsets of $[0,1]$ and the Lebesgue measure $v$, with identification up to $v$-null sets, order convergence coincides with almost everywhere convergence, which is not topological. Furthermore, there are Dedekind complete vector lattices without any Hausdorff vector topology for which every bounded monotone increasing sequence converges to its least upper bound (see also Boccuto and Dimitriou (2015)).

Given a topological space $X$, a nonempty set $\Phi \subset R^{X}$ and a convergence $(\sigma)$ on $\Phi$, we say that $\Phi$ is $(\sigma)$-compact iff every net $\left(f_{\lambda}\right)_{\lambda \in \Lambda}$ in $\Phi$ admits a subnet $\left(f_{\lambda_{k}}\right)_{\kappa \in \Lambda},(\sigma)$-convergent to an element $f \in \Phi$, and that $\Phi$ is $(\sigma)$-closed iff $f \in \Phi$ whenever $\left(f_{\lambda}\right)_{\lambda \in \Lambda}$ is a net in $\Phi,(\sigma)$-convergent to $f \in R^{X}$. The $(\sigma)$-closure of $\Phi$ is the set of the functions $f \in R^{X}$, having a net $\left(f_{\lambda}\right)_{\lambda \in \Lambda}$ in $\Phi(\sigma)$-convergent to $f$. A set $\Phi$ is $(\sigma)$-closed if and only if it coincides with its $(\sigma)$-closure.

We now give the following abstract Ascoli-type theorem. A similar result was given in Boccuto and Dimitriou (2014), Theorem 3.8, where it dealt with uniform convergence on compact sets instead of $(\alpha)$-convergence.

Theorem 3.13. Let $X$ be a Hausdorff topological space, $\mathcal{S}$ and $\mathcal{F}$ be as above.

If $\Phi \subset \Psi \subset R^{X}$, where $\Phi$ is $(\mathcal{S}, \mathcal{F} \alpha)$-closed and $\Psi$ is $(R O \mathcal{F})$-compact, and

$\left.H^{\prime}\right)$ every net $\left(f_{\lambda}\right)_{\lambda \in \Lambda},(R O \mathcal{F})$-convergent in $\Phi$, has a subnet $\left(f_{\lambda_{k}}\right)_{\kappa \in \Lambda},(R O \mathcal{F})$-convergent $\left(\right.$ in $\left.R^{X}\right)$ and $(\mathcal{S}, \mathcal{F})$-exhaustive,

then $\Phi$ is $(\mathcal{S}, \mathcal{F} \alpha)$-compact.

Moreover, if $\Phi$ is $(\mathcal{S}, \mathcal{F} \alpha)$-compact, then $\Phi$ satisfies condition $\left.H^{\prime}\right)$.

Proof: We begin with the first part. Let $\Phi \subset R^{X}$ be $(\mathcal{S}, \mathcal{F} \alpha)$-closed, and $\left(f_{\lambda}\right)_{\lambda \in \Lambda}$ be a net in $\Phi$. Since $\Phi \subset \Psi$ and $\Psi$ is $(R O \mathcal{F})$-compact, $\left(f_{\lambda}\right)_{\lambda \in \Lambda}$ has a $(R O \mathcal{F})$-convergent subnet $\left(f_{\lambda_{k}}\right)_{\kappa \in \Lambda}$. By condition $\left.H^{\prime}\right),\left(f_{\lambda_{k}}\right)_{\kappa \in \Lambda}$ has a subsubnet $\left(f_{\lambda_{k_{\zeta}}}\right)_{\zeta \in \Lambda},(R O \mathcal{F})$-convergent to a function $f \in R^{X}$ and $(\mathcal{S}, \mathcal{F})$-exhaustive. By Theorem 3.2 the net $\left(f_{\lambda_{k_{\zeta}}}\right)_{\zeta \in \Lambda}$ $(\mathcal{S}, \mathcal{F} \alpha)$-converges to $f$. Since $\Phi$ is $(\mathcal{S}, \mathcal{F} \alpha)$-closed, we get $f \in \Phi$. Therefore, $\Phi$ is $(\mathcal{S}, \mathcal{F} \alpha)$-compact.

We now turn to the last part. Let $\Phi$ be $(\mathcal{S}, \mathcal{F} \alpha)$-compact and $\left(f_{\lambda}\right)_{\lambda}$ be a $(R O \mathcal{F})$-convergent net in $\Phi$. Then it admits a subnet $\left(f_{\lambda_{k}}\right)_{\kappa \in \Lambda},(\mathcal{S}, \mathcal{F} \alpha)$-convergent to a function $f \in R^{X}$. By Theorem 3.2, $\left(f_{\lambda_{k}}\right)_{\kappa \in \Lambda}(R O \mathcal{F})$-converges to $f$ and is $(\mathcal{S}, \mathcal{F})$-exhaustive. This ends the proof.

Remarks 3.14. (a) Note that the equicontinuity used in the classical versions of the Ascoli theorem is strictly stronger than property $H^{\prime}$ ) in connection with filter exhaustiveness, even when $R=\mathbb{R}, \mathcal{S}=\mathcal{S}_{\Xi}$ and $\mathcal{F}=\mathcal{F}_{\Lambda}$ (see also Gregoriades and Papanastassiou (2008), Remark 3.2.17). Moreover observe that, in the classical case, the equiboundedness of the involved set $\Phi$ implies the existence of a compact subinterval $[a, b]$ of the real line such that $\Phi$ is contained in the set $[a, b]^{X}$, which is compact with respect to the pointwise topology by virtue of the Tychonoff theorem.

(b) Let $R=\mathbb{R},(p w)$ be the pointwise convergence on $\mathbb{R}^{X}$, and for every $x \in X$ set $\Phi[x]:=\{f(x): f \in \Phi\}$. If $\overline{\Phi[x]}$, the ordinary closure of $\Phi[x]$, is compact in the usual sense, and $\Psi:=\Pi_{x \in X} \overline{\Phi[x]}$, then $\Phi \subset \Psi$ and $\Psi$ is $(p w)$ compact, thanks to the Tychonoff theorem, and so Theorem 3.13 is an extension of Gregoriades and Papanastassiou (2008), Theorems 3.2.19 and 3.2.20. 


\section{Concluding Remarks}

In our setting, a related question, which arises naturally, is to find some necessary and/or sufficient conditions under which the limit function of a suitably pointwise convergent net is constant, or $(\mathcal{S}, \mathcal{F})$-continuous. In this framework, it is advisable to consider the following extensions of the concepts of $(\mathcal{S}, \mathcal{F})$-exhaustiveness and corresponding $(\alpha)$ convergence. We now formulate these notions in connection with continuity; it is possible to argue analogously, if we deal with strong uniform continuity.

Let $X$ be any Hausdorff topological space, $x \in X,(\Xi, \geq)$ and $(\Lambda, \geq)$ be two directed sets, $\mathcal{F}_{1}$ and $\mathcal{F}_{2}$ be two $(\Xi)$-free filters of $\Xi, \mathcal{F}_{3}$ be a $(\Lambda)$-free filter of $\Lambda$, and $f_{\lambda}: X \rightarrow R, \lambda \in \Lambda$, be a function net. We say that $\left(f_{\lambda}\right)_{\lambda}$ is $\left(\mathcal{F}_{1}, \mathcal{F}_{2}, \mathcal{F}_{3}\right)$ exhaustive at $x \in X$ iff there exists an $(O)$-sequence $\left(\sigma_{p}\right)_{p}$ in $R$ such that, for every net $x_{\xi}, \xi \in \Xi, \mathcal{F}_{1}$-convergent to $x$ and for any $p \in \mathbb{N}$ there are $F_{2} \in \mathcal{F}_{2}$ and $F_{3} \in \mathcal{F}_{3}$ with $\left|f_{\lambda}\left(x_{\xi}\right)-f_{\lambda}(x)\right| \leq \sigma_{p}$ for every $\xi \in F_{2}$ and $\lambda \in F_{3}$. The net $f_{\lambda}: X \rightarrow R, \lambda \in \Lambda$, is weakly $\left(\mathcal{F}_{1}, \mathcal{F}_{2}, \mathcal{F}_{3}\right)$-exhaustive at $x \in X$ iff there is an $(O)$-sequence $\left(\sigma_{p}\right)_{p}$ in $R$ such that, for each net $x_{\xi}, \xi \in \Xi, \mathcal{F}_{1}$-convergent to $x$ and for every $p \in \mathbb{N}$ there is a set $F_{2} \in \mathcal{F}_{2}$ such that for each $\xi \in F_{2}$ there exists $F_{\xi} \in \mathcal{F}_{3}$ with $\left|f_{\lambda}\left(x_{\xi}\right)-f_{\lambda}(x)\right| \leq \sigma_{p}$ whenever $\lambda \in F_{\xi}$.

The net $f_{\lambda}: X \rightarrow R, \lambda \in \Lambda,\left(\mathcal{F}_{1}, \mathcal{F}_{2}, \mathcal{F}_{3} \alpha\right)$-converges to $f: X \rightarrow R$ at $x \in X$ iff there exists an $(O)$-sequence $\left(\sigma_{p}\right)_{p}$ in $R$ such that, for every net $x_{\xi}, \xi \in \Xi, \mathcal{F}_{1}$-convergent to $x$ and for each $p \in \mathbb{N}$ there are $F_{2} \in \mathcal{F}_{2}, F_{3} \in \mathcal{F}_{3}$ with $\left|f_{\lambda}\left(x_{\xi}\right)-f(x)\right| \leq \sigma_{p}$ whenever $\xi \in F_{2}$ and $\lambda \in F_{3}$.

With the same techniques as in the previous sections, it is possible to prove the following characterization of (weak) filter exhaustiveness in terms of filter $(\alpha)$-convergence and filter continuity of the limit function.

Theorem 4.1. Let $f_{\lambda}: X \rightarrow R, \lambda \in \Lambda$, be a net of functions, $\left(R O \mathcal{F}_{3}\right)$-convergent to $f: X \rightarrow R$, and $x \in X$. Then $\left(f_{\lambda}\right)_{\lambda}$ is $\left(\mathcal{F}_{1}, \mathcal{F}_{2}, \mathcal{F}_{3} \alpha\right)$-convergent to $f$ at $x$ if and only if $\left(f_{\lambda}\right)_{\lambda}$ is $\left(\mathcal{F}_{1}, \mathcal{F}_{2}, \mathcal{F}_{3}\right)$-exhaustive at $x$. Moreover, $\left(f_{\lambda}\right)_{\lambda}$ is weakly $\left(\mathcal{F}_{1}, \mathcal{F}_{2}, \mathcal{F}_{3}\right)$-exhaustive at $x$ if and only if $f$ is $\left(\mathcal{F}_{1}, \mathcal{F}_{2}\right)$-continuous at $x$.

Acknowledgement: Our thanks to Prof. W. Wilczyński for carefully reading the manuscript and suggesting some modifications which improved the exposition of the paper.

Open problems: (a) Prove similar results for sequences/nets of functions/measures with values in some other abstract structures and/or with respect to other kinds of convergence.

(b) Establish some other comparisons/relations between different types of (strong weak) filter exhaustiveness for nets of functions/measures (see also Boccuto and Dimitriou (2015)).

\section{References}

Athanassiadou, E., Boccuto, A., Dimitriou, X., \& Papanastassiou, N. (2012). Ascoli-type theorems and ideal $(\alpha)$-convergence. Filomat, 26(2), 397-405. http://dx.doi.org/10.2298/FIL1202397A

Athanassiadou, E., Dimitriou, X., Papachristodoulos, Ch., \& Papanastassiou, N. (2012). Strong ( $\alpha$ )-convergence and ideal strong exhaustiveness of sequences of functions. Int. J. Pure Appl. Math., 80(2), 207-216.

Baláž, V., Červeňanskij, J., Kostyrko, P., \& Šalát, T. (2002). I -convergence and $\mathcal{I}$-continuity of real functions. Acta Math. (Nitra), 5, 43-50.

Beer, G., \& Levi, S. (2009). Strong uniform continuity. J. Math. Anal. Appl., 350, 568-589.

Boccuto, A., Das, P., Dimitriou, X., \& Papanastassiou, N. (2012). Ideal exhaustiveness, weak convergence and weak compactness in Banach spaces. Real Anal. Exchange, 37(2), 389-410.

Boccuto, A., \& Dimitriou, X. (2011). Some properties of ideal $\alpha$-convergence in ( $\ell$ )-groups. Int. J. Pure Appl. Math., 72(1), 93-99.

Boccuto, A., \& Dimitriou, X. (2014). Ascoli-type theorems in the cone metric space setting. J. Inequalities Appl. Art., 420, 16.

Boccuto, A., \& Dimitriou, X. (2015). Convergence Theorems for Lattice Group-Valued Measures. Bentham Science Publ., Sharjah, U. E. A. ISBN 9781681080109.

Boccuto, A., Dimitriou, X., \& Papanastassiou, N. (2012a). Basic matrix theorems for $\mathcal{I}$-convergence in $(\ell)$-groups. Math. Slovaca, 62(5), 885-908. http://dx.doi.org/10.2478/s12175-012-0053-6

Boccuto, A., Dimitriou, X., \& Papanastassiou, N. (2012b). Schur lemma and limit theorems in lattice groups with 
respect to filters. Math. Slovaca, 62(6), 1145-1166. http://dx.doi.org/10.2478/s12175-012-0070-5

Boccuto, A., Dimitriou, X., Papanastassiou, N., \& Wilczyński, W. (2011). Ideal exhaustiveness, continuity and $(\alpha)$-convergence for lattice group-valued functions. Int. J. Pure Appl. Math., 70(2), 211-227.

Boccuto, A., Dimitriou, X., Papanastassiou, N., \& Wilczyński, W. (2014). Modes of ideal continuity and the additive property in the Riesz space setting. J. Appl. Anal., 20(1), 41-53.

Candeloro, D., \& Sambucini, A. R. (2015). Filter convergence and decompositions for vector lattice-valued measures. Mediterranean J. Math, in press. http://dx.doi.org/10.1007/s00009-014-0431-0

Carathéodory, C. (1929). Stetige Konvergenz und normale Familien von Funktionen. Math. Ann., 101, 515-533.

Caserta, A., Di Maio, G., \& Holá, L. (2010). Arzelà’s theorem and strong uniform convergence on bornologies. J. Math Anal. Appl., 371, 384-392.

Caserta, A., Di Maio, G., \& Kočinac, Lj. D. R. (2011). Statistical convergence in function spaces. Abstr. Appl. Anal., 2011, Article ID420419, 11 pages.

Gregoriades, V., \& Papanastassiou, N. (2008). The notion of exhaustiveness and Ascoli-type theorems. Topology Appl., 155, 1111-1128.

Hahn, H. (1922). Über Folgen linearer Operationen. Monatshefte für Math. und Physik, 32, 3-88.

Kelley, J. L. (1955). General Topology. D. Van Nostrand Co., Inc., Princeton.

Mačaj, M., \& Sleziak, M. (2011). $\mathcal{I}^{\mathcal{K}}$-convergence. Real Anal. Exchange, 36(1), 177-194.

Sleziak, M. (2003). I-continuity in topological spaces. Acta Math. (Nitra), 6, 115-122.

Zygmund, A. (1968). Trigonometric series, Vol. I. Cambridge Univ. Press, Cambridge.

\section{Copyrights}

Copyright for this article is retained by the author(s), with first publication rights granted to the journal.

This is an open-access article distributed under the terms and conditions of the Creative Commons Attribution license (http://creativecommons.org/licenses/by/3.0/). 